\title{
Imagen corporal. Un estudio comparativo entre hombres homosexuales y heterosexuales
}

\author{
Body image. A Comparative Study between Homosexual and Heterosexual Men
}

\author{
Rebeca Guzmán-Saldaña ${ }^{1}$, Gilda Gómez-Peresmitré ${ }^{2}$, Rodrigo León-Hernández ${ }^{3}$, Silvia \\ Platas-Acevedo ${ }^{4}$, Karina Reyes-Jarquín ${ }^{5}$
}

\begin{abstract}
:
A comparative study was carried out between homosexual and heterosexual men, in order to establish differences regarding the evaluation of body image and risky eating behaviors. A design of two samples with independent observations was used. The total non-random sample of $\mathrm{N}=32$ men with an age range of 20 to 45 years was subdivided with $\mathrm{n}_{1}=11$ heterosexual men $(M=28.9, S D$ $=8.77)$ and $\mathrm{n}_{2}=21$ homosexual men $(M=23.89, S D=3.77)$. To evaluate the body image, the Silhouettes Visual Scale was applied, likewise, the Attitude Scale was applied to the Male Body. Also, a survey of sociodemographic data. No significant differences were found between the two samples of participants regarding the perception of body image, both show dissatisfaction, approximately half are perceived with obesity and want to be thinner and more robust (especially in heterosexuals). There is a slight tendency related to a higher percentage of homosexual men who want to be thinner (positive dissatisfaction), compared to that of heterosexual men.
\end{abstract}

Keywords:

Homosexual Men, Heterosexual Men, Body Image

\section{Resumen:}

Se realizó un estudio comparativo entre hombres homosexuales y heterosexuales, con la finalidad de establecer diferencias respecto a la evaluación de la imagen corporal y conductas alimentarias de riesgo. Se utilizó un diseño de dos muestras con observaciones independientes. La muestra total no aleatoria de $\mathrm{N}=32$ hombres con un rango de edad de 20 a 45 años, quedó subdividida con $\mathrm{n}_{1}=11$ hombres heterosexuales $(\mathrm{M}=28.9, \mathrm{DE}=8.77) \mathrm{y}_{2}=21$ hombres homosexuales $(\mathrm{M}=23.89, \mathrm{DE}=3.77)$. Para evaluar la imagen corporal se aplicó la Escala Visual de Siluetas, así mismo, se aplicó la Escala de Actitudes hacia el Cuerpo Masculino. También una Encuesta de datos sociodemográficos. No se encontraron diferencias significativas entre las dos muestras de participantes en cuanto a la percepción de la imagen corporal, ambos muestran insatisfacción, aproximadamente la mitad se perciben con obesidad y desean ser más delgados y robustos (sobre todo en los heterosexuales). se mantiene una ligera tendencia relacionada con un mayor porcentaje de hombres homosexuales que quiere estar más delgado (insatisfacción positiva), comparado con el de hombres heterosexuales.

Palabras Clave:

Hombres Homosexuales, Hombres Heterosexuales, Imagen Corporal

\section{Introducción}

Para iniciar, es importante precisar que este reporte es resultado de una investigación en línea (online) en la que se compararon datos de hombres homosexuales contra hombres heterosexuales respecto a variables

\footnotetext{
${ }^{1}$ Universidad Nacional Autónoma de México. Facultad de Psicología. Autor de Correspondencia: Universidad Autónoma del Estado de Hidalgo. Instituto de Ciencias de La Salud. Área Académica de Psicología.correo: rguzman@uaeh.edu.mx. https://orcid.org/0000-0003$0877-4871$.

${ }^{2}$ Universidad Nacional Autónoma de México. Facultad de Psicología. Gilda Gómez-Peresmitré, correo: gildag @unam.mx

${ }^{3}$ Universidad Nacional Autónoma de México. Facultad de Psicología. Silvia Platas-Acevedo, correo: romsip@uanm.mx

${ }^{4}$ Universidad Nacional Autónoma de México. Facultad de Psicología. Rodrigo León-Hernández, correo: rod_leonher@yahoo.com.mx

${ }^{5}$ Universidad Nacional Autónoma de México. Facultad de Psicología. Karina Reyes-Jarquín, correo: karina_reyes@uaeh.edu.mx
} 
relacionadas con la imagen corporal.

Ya adentrándonos en la temática, la figura ideal de los varones es diferente a la de las mujeres, para quienes, el deseo de un modelo corporal va acompañado por la idea "a más delgada mejor" (Furnham, Badmin, \& Sneade, 2002). En los hombres como género, puede afirmarse como una primera tendencia, que la figura ideal dominante, es mesomorfa, gruesa, y por ende con músculo y sin grasa (Gómez-Peresmitré, Granados, Jáuregui, Tafoya, \& Unikel, 2000; Gómez-Peresmitré, Jaeger, Pineda, \& Platas, 2010), ellos desean mayoritariamente una figura atlética con más músculo, hombros y espalda ancha y cintura delgada; es decir, con aumento de masa y tono muscular (Carlson \& Crawford, 2005; McCabe \& Ricciardelli, 2001; 2003; McCrary \& Sasse, 2000; Raevouri, KeskiRahkonen, Bulik, Rose, Aila, \& Kapric, 2006).

En México, diversos estudios realizados en niños, púberes y adolescentes hombres señalan que casi la mitad de los participantes desean tener un cuerpo más grueso del que tienen, con una figura atlética (Gómez-Peresmitré et al., 2000; GómezPeresmitré, Alvarado, Moreno, Saloma, \& Pineda, 2001). Entre los hombres adultos como lo señalan Gray y Ginsberg (2007), se ha incrementado en las últimas décadas la proporción de personas insatisfechas con su cuerpo (peso y forma). Sin embargo, hay una segunda tendencia, en donde se encuentra una minoría de hombres que al igual que las mujeres quieren una figura delgada, y se ha encontrado que este deseo de delgadez se encuentra asociado con trastornos de la conducta alimentaria (TCA), Anorexia Nervosa (AN), Bulimia Nervosa (BN); y con la orientación sexual dirigida hacia el mismo sexo, reportando que entre los hombres homosexuales 0 bisexuales existe una propensión a presentar un índice más alto de TCA que entre los hombres heterosexuales (Feldman \& Meyer, 2007; Lazo, 2012).

Se señala que el rango de hombres homosexuales o bisexuales con AN es de un $20-45 \%$ (Russell \& Keel, 2002). Asimismo, en una investigación con estudiantes universitarios latinoamericanos, se observó que entre los hombres con algún TCA, un 58\%, eran homosexuales (Toro, Nieves, \& Borrero, 2010). La gran prevalencia de hombres homosexuales con TCA sugiere que éstos tienen una mayor susceptibilidad a desarrollarlos (Do Jackson, 2008; Hospers \& Jensen, 2005). Hay evidencia de que los hombres con BN tienen una más alta prevalencia de homosexualidad y que ésta actúa como factor de riesgo para la bulimia en hombres, pero no en mujeres (Carlat \& Camargo, 1991).

Podría señalarse que la orientación sexual homosexual tiene un papel predisponente en varones, se ha encontrado que los TCA tienen mayor prevalencia en hombres homosexuales (Hospers \&
Jansen, 2005). Sin embargo, al igual que entre los heterosexuales, entre los hombres homosexuales hay quienes quieren una figura ideal delgada y quienes desean una figura esbelta, pero musculada (Siever, 1994); figura que, de acuerdo con Wood (2004), es altamente valorada en la comunidad gay. Debe tomarse en cuenta que para el homosexual masculino la apariencia tiene un valor apreciado, igual que en el mundo femenino (Wichstrom, 2006).

En los estudios realizados entre estudiantes masculinos universitarios en los que se comparan homosexuales contra heterosexuales, se ha encontrado que las diferencias entre estos grupos pueden deberse al énfasis que los primeros otorgan a la apariencia. Los hombres homosexuales muestran mayor insatisfacción corporal. El ejercicio está más motivado por el deseo de mejorar el atractivo corporal, valoran más la apariencia corporal, puntúan significativamente más bajo en la escalas de musculatura (Silberstein, Mishkind, Striegel-Moore, Timko, \& Rodin, 1989; Siever, 1994); Silberstein y colaboradores (1989), reportaron, además, que en el grupo de hombres homosexuales pero no en el de heterosexuales, los hombres que deseaban ser más delgados tenían más actitudes y conductas asociadas con conducta alimentaria anómala que los hombres que eran más delgados de lo que ellos deseaban. Asimismo, los homosexuales manifiestaban más síntomas depresivos, ansiedad general, fobias y trastornos psiquiátricos (Feldman \& Meyer, 2007).

¿Pero la apariencia entre los hombres es en verdad atractiva sólo para los hombres homosexuales? En la actualidad, la postmodernidad (pérdida de grandes números de trabajadores de cuello azul, por ejemplo de obreros, el crecimiento de una economía de servicios, la expansión de las competencias y habilidades femeninas a prácticamente todos los campos del saber, hacer y decir, etc.) va acompañada de una cada vez mayor atención por la presentación, por la apariencia, tendencia que ha coincidido con el surgimiento de una figura "postmodernizada" de un hombre pulcro, "femenizado" en su presentación, preocupado por su cara (tratamientos de belleza) ropa, cabello, uñas, etc., y también preocupado por la forma de su cuerpo (esto es acuden a la cirugía estética, cuyas cifras y modalidades pueden consultarse en cualquiera de las estadísticas que existen al respecto). Puede decirse, entonces, que ha surgido una nueva figura. Se habla del hombre "metrosexual" y del "ubersexual", que no son necesariamente homosexuales (Rey, 1994; Pérez-Gauli, 2000).

De ahí que la atracción por la apariencia no se encuentra solo ligada con la homosexualidad, ya que entre los heterosexuales existen grupos de hombres que también se sienten atraídos por la apariencia ¿Con qué prevalencia?, ¿bajo qué condiciones? Es claro que las respuestas requieren investigación. Con respecto a la figura ideal dominante, se tienen datos 
recientes de una investigación en universitarios que dejaron ver que el $73 \%$ de los hombres heterosexuales mostraban una alta adherencia a esa figura (cuyos atributos representan fuerza física, musculatura, virilidad, agresividad e independencia) (Toro, Nieves, \& Borrero, 2010).

Ahora bien, una imagen corporal negativa constituye un factor de riesgo para el desarrollo no sólo de los TCA, sino también de otras patologías que atentan contra la salud mental (Knoesen, Thai Vo, \& Castle, 2009). Los efectos dañinos de una pobre imagen corporal se han comparado con los de desadaptación social y se muestran en la forma de patología alimentaria (GómezPeresmitré et al., 2000, 2010) en insatisfacción y distorsión corporal (Moreno \& Ortiz, 2009; Vázquez, et al., 2005) y en la forma de pensamientos y sentimientos negativos dirigidos al propio cuerpo (Grogan, 2008).

Con base en los antecedentes descritos y teniendo como propósito central de la investigación la exploración de la imagen corporal masculina, se realizó un estudio que tuvo como objetivo describir las muestras analizando las principales variables sociodemográficas; y contrastar la imagen corporal entre los participantes con diferente orientación sexual, determinando la existencia de diferencias significativas con respecto a imagen corporal (insatisfacción corporal y figura ideal).

\section{Método}

Definición de variable

\section{Imagen corporal}

Es la configuración global, o conjunto de representaciones, percepciones, sentimientos y actitudes, que el individuo elabora con respecto a su cuerpo durante su existencia, a través de diversas experiencias (Bruchon-Schweitzer, 1992).

Tipo de Estudio: Descriptivo, transversal, no experimental.

Muestra y participantes: La investigación se ajustó a un diseño de dos muestras con observaciones independientes. La muestra total no aleatoria de $\mathrm{N}=32$ hombres con un rango de edad de 20 a 45 años, quedó subdividida con $\mathrm{n} 1=11$ hombres heterosexuales $(M=28.9, D E=8.77)$ y $\mathrm{n} 2=21$ hombres homosexuales $(M=23.89, D E=3.77)$.

\section{Instrumentos}

Se aplicó un Cuestionario sociodemográfico con 22 preguntas formato mixto (abiertas, cerradas, dicotómicas y de opción múltiple).

Para la medición de imagen corporal se aplicó la Escala visual de siluetas (Gómez-Peresmitré, 2010). Las variables medidas fueron:
Satisfacción/insatisfacción que son factores de la Escala de Insatisfacción con Partes Corporales (Gómez-Peresmitré, 2011). Así mismo, se aplicó la Escala de actitudes hacia el cuerpo masculino (Male Body Actittude Scale) (Tylka, Bergeron, \& Schwartz, 2005) que tiene una estructura factorial y consistencia interna obtenidas en muestras de hombres mexicanos (Gómez-Peresmitré, 2011). Es importante destacar que la aplicación de los instrumentos se realizó a través de internet.

\section{Resultados}

\section{Datos sociodemográficos}

En cuanto a la descripción sociodemográfica de las muestras, el lugar de residencia de los participantes la mayoría (62\%) vive en la Ciudad de México, mientras que el $22 \%$ en el Estado de México, en el estado de Hidalgo, y en el de Veracruz residía el resto de los que respondieron por Internet al instrumento del presente estudio.

Los Hombres Heterosexuales (hhet) tenían en su totalidad niveles significativamente más altos de estudios $(p=0.05)$ de licenciatura $(91 \%)$ y de posgrado (9\%); mientras que el $29 \%$ de los Hombres Homosexuales (hhom) tenían preparatoria y el 62\% licenciatura. Los hhet eran en su gran mayoría solteros (hhet, 90\%) mientras que los hhom lo eran en su totalidad (hhom, 100\%); sólo un $10 \%$ de los primeros eran casados. Más hhet trabajaban (50\%) comparados con el $33 \%$ de hhom que también lo hacían. Cuando se preguntó por la ocupación, el mayor porcentaje en los dos grupos fue la de estudiante (hhet $=73 \%$ vs. hhom $=76 \%$ ); los porcentajes de empleado (19\%) y profesionista (18\%) fueron los que siguieron y correspondieron a los hhet y hhom respectivamente. Ninguno de los hhet así como ninguno de los hhom aceptó consumir esteroides.

\section{Escala de Siluetas}

Imagen corporal: A. ¿Cómo se auto-percibían (figura actual o real) los hombres de las muestras de este estudio? Un $45 \%$ de los hhet y $29 \%$ de los hhom se vieron a sí mismos obesos (ver silueta 8); y otro $5 \%$ de estos últimos eligió la silueta 9; más hhom se autopercibieron con sobrepeso: siluetas (6) $19 \%$ y (7) $9 \%$; en comparación con el $18 \%$ de los hhet que seleccionó la silueta (6). Por otra parte, el $29 \%$ de hhom y el $9 \%$ de hhet se autopercibieron con normopeso (silueta 5). El $9 \%$ de hhom y el 18\% de hhet se vieron excesivamente delgados (silueta 1 ) y el $10 \%$ de hhet seleccionó la silueta 2, muy delgados.

B. ¿Cuál era su figura ideal? Las respuestas del $100 \%$ de los hhet se distribuyeron como sigue: para la silueta (4) que corresponde a delgadez con músculo con un 
$46 \%$; y siluetas (6) con $27 \%$ y (7) con $27 \%$ correspondientes a sobrepeso ligero y a sobrepeso. Entre los hhom, el 38\% seleccionó como figura ideal una silueta delgada con músculo (4), un $5 \%$ la silueta con sobrepeso ligero (6) y otro $38 \%$ la silueta (7) con sobrepeso. Entre los hhom el $10 \%$ seleccionó una silueta excesivamente delgada (1) y un $9 \%$ una muy delgada, silueta (2).

C. ¿Cómo era su insatisfacción corporal? Al respecto las respuestas de la variable "insatisfacción corporal" según la orientación sexual, el $62 \%$ de los hhom vs. $54 \%$ de los hhet mostraron estar insatisfechos porque deseaban estar más delgados (insatisfacción positiva). Los niveles más altos de insatisfacción fueron los de los hhom que se encuentran en los niveles 5 y 7 mientras que el nivel de insatisfacción más alto de los hhet se encuentra en el 4. Entre más grande es la diferencia entre figura actual y figura ideal, mayor es la insatisfacción y que la no diferencia entre estas figuras se interpreta como satisfacción.

En cambio, sólo un $45 \%$ de los hhet y un 33\% de los hhom tenían insatisfacción negativa, esto es, deseaban un cuerpo más robusto. Sin embargo, este tipo de insatisfacción era más fuerte $(-5)$ en un $18 \%$ de los hhet que en los hhom (5\% con nivel -3$)$. Por otra parte, llama la atención que sólo un $5 \%$ de hhom se encuentre satisfecho y ninguno de los hhet.

Con respecto al IMC se encontró que los hhet tenían porcentajes más altos de sobrepeso y obesidad que los hhom y que estos últimos tenían el porcentaje más alto de peso normal (62\%) en comparación al 36\% de los hhet.

\section{Factores de insatisfacción con partes corporales (eipc)}

Con respecto a la variable "insatisfacción con partes corporales" se encontró en relación al Factor 1 (estómago, cintura y cadera) que prácticamente el mismo porcentaje de hhom y hhet (33\% vs $30 \%$ ) respectivamente tenían mayor insatisfacción positiva (querían partes corporales más delgadas) que negativa, partes corporales más gruesas (14\% vs. $22 \%$ ) respectivamente, sin que esas diferencias fueran estadísticamente significativas. Los porcentajes más altos fueron para satisfacción, hhom $52 \%$ y hhet $47 \%$.

En el Factor 2 (espalda, brazos y tórax) tampoco se encontraron diferencias entre hhom y hhet y las respuestas, dentro de este último grupo se dividieron en partes iguales (33.3\%) para insatisfacción positiva, satisfacción e insatisfacción negativa; para los hhom la división fue muy similar (30\%, 40\%, 30\%); por último, en relación con el Factor 3 (estatura, glúteos, muslos y pantorrillas) en los dos grupos se aprecian porcentajes similares, siendo un poco más altos los de insatisfacción positiva hhom $(43 \%)$ y hhet $(45 \%)$.

\section{Factores obtenidos con la Escala de Actitud hacia el Cuerpo Masculino de Tylka, Bergeron y Schwartz (2005)}

Ninguno de los factores: Factor 1, preocupación por la grasa, tamaño y forma corporal; Factor 2, insatisfacción con tamaño, definición y músculo y Factor 3, satisfacción corporal, comparados por grupo (orientación sexual) arrojó resultados estadísticamente significativos. La subdivisión en percentiles de las respuestas de cada factor mostró con respecto al Factor 1 que el $18 \%$ de los hhet tenía una "preocupación severa" comparado con la de casi el doble (30\%) de los hhom; los porcentajes más altos fueron para "preocupación leve" (hhet $45 \%$ vs. 35\% hhom). Con respecto al Factor 2 se encontró insatisfacción severa en porcentajes similares a los del Factor 1 (hhet $18 \%$ vs. $26 \%$ hhom); lo mismo puede decirse para insatisfacción leve (hhet 45\% y 32\% hhom) y moderada (hhet $36 \%$ vs. $42 \%$ hhom). Por último, en relación a satisfacción corporal sobresale el alto porcentaje (64\%) de hhet con baja satisfacción corporal comparado con el $35 \%$ de los hhom; un $27 \%$ de los hhet se encontraba con alta satisfacción corporal en comparación con el 30\% de los hhom.

\section{Discusión y Conclusiones}

Los grupos de hhom y hhet quedaron formados, en su gran mayoría, por estudiantes, aunque más hhet que hhom respondieron que también trabajaban. Los hhet casi en su totalidad (99\%) tenía estudios de licenciatura y posgrado, mientras que entre los hhom un poco más de una cuarta parte de ellos, estudiaba preparatoria y, casi dos tercios, licenciatura. La totalidad de los hhom era soltero y sólo una décima parte de los hhet eran casados, por lo que prácticamente los dos grupos estaban formados por hombres solteros.

Sorprende encontrar que a una edad tan joven, antes de los 10 años, más de un tercio de la muestra de los hhom se dio cuenta que lo que lo atraía sexualmente, su orientación sexual, "no era", no se ajustaba, a lo que los otros esperaban; sumado a otro tercio de la muestra, puede decirse que una gran mayoría de los hombres de este grupo se dio cuenta de su homosexualidad entre los 10 y 15 años, y la edad en la que la revelaron fue similar entre los 11 y 15 años. Se tiene que pensar que esta edad (pubertad y adolescencia temprana) trae consigo una serie de ajustes cognoscitivos, conductuales, emocionales y biológicos, que en general causan problemas a los chicos. A todo esto, habría que agregar lo que trae aparejado la homosexualidad en una sociedad machista como la mexicana. 
Un programa orientado a mejorar la calidad de vida de los chicos en esta etapa etaria debe proponerse, entre otros objetivos, la detección de factores de riesgo asociados con la orientación sexual (heterosexual y homosexual) para trabajar con los chicos y con los padres, pues, en el caso de los homosexuales, éstos, los padres, requieren reeducarse en oposición a la cultura homofóbica en la que vivimos inmersos e inconscientes y sensibilizarse a las necesidades (de afecto, seguridad y autoestima, entre otras) de sus hijos.

Resulta interesante conocer que el $100 \%$ de los hhet escogería, ser heterosexual (si esta actividad fuera posible en algún momento de la vida), mientras que entre los hhom se encontró que un $5 \%$ elegiría ser heterosexual y otro $5 \%$ bisexual. La mayoría de los hombres de los dos grupos vive con su familia. Los hhom tienen más amigos cercanos que los hhet. Así, los hhom tienen un porcentaje alto y mayor de amigos (3 a 5 y más de 5) que los hhet y un 10\% de éstos, también respondieron tener ningún amigo comparado con un $0 \%$ de los hhom. Un porcentaje más alto de hhet calificó sus relaciones familiares como "buenas" y "muy buenas" y más hhom las calificó como "regulares".

Asimismo, la gran mayoría de los hombres de los dos grupos respondió no consumir esteroides ni otras drogas, ya que sólo un $15 \%$ de hhom y un $9 \%$ de hhet aceptaron consumirlas. Más heterosexuales consumían tabaco y bebidas alcohólicas, aunque curiosamente también hacían más deporte o ejercicio que los hhom.

Las muestras de este estudio son prácticamente de estudiantes y con niveles altos de estudios, especialmente los hhet. Asimismo, en este estudio se encontró que la muestra de heterosexuales es una muestra que confirma la vigencia del problema de salud pública en México (ver ENSANUT, 2012), puesto que resultó con sobrepeso (36\%) y obesidad (18\%) de acuerdo con los puntos de corte de la OMS (2000); aunque entre los hhom hubo un $24 \%$ de sobrepeso, el porcentaje de obesidad fue bajo (5\%).

Ahora bien, con respecto a imagen corporal, se encontró que la gran mayoría de los hhet y de los hhom, dos tercios de las muestras, se autopercibieron (recuérdese que autopercepción es una variable de carácter subjetivo) con sobrepeso y obesidad, esto es, sobreestimaron su peso, cuando en realidad y de acuerdo con su IMC el porcentaje de los hhet con sobrepeso y obesidad era menor y todavía más bajos eran los porcentajes de los hhom. Asimismo, un poco más de una cuarta parte de los hhet se vieron a sí mismos muy delgados cuando menos de un $10 \%$ de ellos tenía un IMC equivalente a delgadez, y curiosamente la autopercepción de delgadez de los hhom coincidió exactamente con el IMC correspondiente de delgadez.

Con respecto a normopeso, un poco menos de la tercera parte de los hhom se vieron a sí mismos con normopeso cuando, de acuerdo con su IMC, casi el doble de ellos tenía efectivamente peso normal y, al contrario, un pequeño porcentaje de hhet (menos del $10 \%$ ) se vieron con peso normal, cuando más de un tercio de ellos tenían IMC correspondiente a peso normal. Ninguno de los participantes de los dos grupos eligió las figuras 8 y 9 representantes de obesidad, lo que coincidió con las respuestas de los participantes del primer estudio.

La figura ideal para los hhet fue, por haber sido elegida con la mayor frecuencia, la de la silueta 4 (delgada con músculo), mientras que los hhom seleccionaron dos siluetas con la misma frecuencia, la 4 (delgadez con músculo) y la 7 (sobrepeso ligero), aunque esta última también fue elegida, en segundo lugar, por los hhet. Asimismo, sólo los hhom eligieron la silueta extremadamente delgada y la muy delgada. Es importante destacar que no fueron elegidas las siluetas representantes de obesidad, esto confirma la tendencia que señala que los hhom quieren estar delgados (Do Jackson, 2008; Feldman \& Meyer, 2007; Siever, 1994).

Por otra parte, no debe perderse de vista que las muestras de este estudio tenían porcentajes altos de sobrepeso y obesidad en especial los hhet que los hhet. Sin embargo, la figura ideal, tanto de los hhom como la de los hhet es similar (delgada, con músculo o sobrepeso ligero), sin importar la orientación sexual, pues ninguna de las variables relacionadas con imagen corporal (figura actual, figura ideal $e$ insatisfacción) resultaron estadísticamente significativas, lo que también es coincidente con los resultados del primer estudio.

Estos resultados van en contra de la teoría, ya que se asume que los hombres se encuentran menos insatisfechos o más satisfechos que las mujeres porque no desean estar más delgados. Sin embargo, aunque no hay diferencia significativa que permita afirmar que son desiguales, se mantiene una ligera tendencia relacionada con un mayor porcentaje de hhom que quiere estar más delgado (insatisfacción positiva) comparado con el de hhet o viceversa el mayor porcentaje de los hhet comparado con el de los hhom que quiere un cuerpo más robusto (insatisfacción negativa) respectivamente.

Asimismo, con respecto a insatisfacción con partes corporales se encontraron respuestas similares en los dos grupos. Esto es no se encontraron diferencias significativas en ninguno de los tres factores que componen la escala que estima insatisfacción con partes corporales. La actitud hacia el cuerpo masculino puede decirse que prácticamente fue la misma para los hhom como para los hhet, mostrando similar preocupación por grasa, tamaño y forma corporal e insatisfacción con tamaño, definición y músculo, sin importar la orientación sexual. No obstante, la distribución de los porcentajes de "preocupación severa" con un porcentaje mayor de hhom marca una tendencia coincidente con resultados 
del primer estudio en el que se encontró una diferencia significativa en este Factor 1, mostrando que eran los hhom quienes se preocupaban más.

Por otra parte, fue notorio que una mayoría de hhet, casi dos tercios, tuviera baja satisfacción corporal. Sin embargo, si se analizan los resultados a la luz de los obtenidos en autopercepción de la figura actual (figura real) puede verse que casi tres cuartas partes de hhet se autopercibió obeso y con sobrepeso. Y si a esto se añaden los resultados del IMC que indican altos porcentajes de sobrepeso en hhom y hhet $y$ altos porcentajes de obesidad entre los hhet pueden entenderse mejor los resultados de satisfacción corporal.

Entre las conclusiones más importantes se encuentran los hallazgos comunes a los dos estudios:

1. Figura ideal correspondiente a una silueta delgada con músculo y a una silueta con sobrepeso ligero.

2. La gran mayoría de los participantes estaban insatisfechos porque querían una figura más gruesa, independientemente de su orientación sexual.

3. Porcentajes bajos de satisfacción corporal.

4. Mayor preocupación de los hhom por grasa, tamaño y forma corporal que los hhet.

Por último, puede decirse que los resultados de imagen corporal (figura actual, figura ideal, e insatisfacción corporal) un tanto inconsistentes con estudios previos y consistentes intra-variables suele ser lo más frecuente en los inicios de cualquier campo de estudio que, como estamos viendo, es lo que sucede con los datos provenientes de los factores de riesgo en hombres, lo que no es más que una señal inequívoca de la necesidad de mayor investigación. Debe señalarse que una de las limitaciones de mayor importancia en este estudio es el tamaño pequeño de las muestras, de aquí la necesidad de investigar más en torno a imagen corporal, tomando en cuenta la orientación sexual y cuidando el tamaño de las muestras.

\section{Referencia}

Boon, S. D. \& Lomore, C. D. (2001). Admirer-celebrity relationships among young adults: explaining perceptions of celebrity influence on identity. Human Communication Research, 27, 432-465.

Bruchon-Schweitzer, M. (1992). Psicología del cuerpo. Barcelona: Herder

Do Jackson, C. (2008). Exploration of Factors Associated with Eating Disorders in Gay

Men. Doctoral dissertation (n/p). Disponible: 〈www.Ir.Iowa.edu〉.

Feldman, M. \& Meyer, I. (2007). Eating disorders in diverse lesbian, gay and bisexual

Populations. International Journal of Eating Disorders, 40, 218-26.

Gómez-Peresmitré, G. (online). Diversidad sexual e imagen corporal. $<$ http://servicios.psicol.unam.mx/diversidadsexual/>.
Gómez-Peresmitré, G. (2011). Imagen corporal. ¿Qué quieren los hombres? Conferencia

Magistral, Ciclo de Investigación en Psicología, Facultad de Psicología, UNAM.

Organización Mundial de la Salud (2000). Informe sobre la salud en el mundo 2000.

Mejorar el desempeño de los sistemas de salud. [Recuperado el 10 de mayo de 2008]. <http://www. who.int/whr/2000/es/>.

Pérez-Gauli, J. (2000). El cuerpo en venta. Relación entre arte y publicidad. Madrid: Ediciones.

Rey, J. (1994). El hombre fingido. La representación de la masculinidad en el discurso Publicitario. España: Fundamentos.

Siever, M. (1994). Sexual orientation and gender as factors in socioculturally acquired vulnerability to body dissatisfaction and eating disorders. Journal of Consulting and Clinical Psychology, 62, 252-260.

Tylka, T. L., Bergeron, D. \& Schwartz, J. P. (2005). Development and psychometric evaluation of the male body attitudes scale (MBAS). Body Image, 2, 161-175.

Toro, J., Nieves, L. \& Borrero, N. (2010). Cuerpo y masculinidad: los desórdenes alimentarios en hombres. Interamerican Journal of Psychology, 44, 225-234

Wichstrom, L. (2006). Sexual orientation as a risk factor for bulimic symptoms. International Journal of Eating Disorders, 39, 448-453.

Wood, M. (2004). Body image disturbance and gender oppression among gay men, Journal of Gay and Lesbian Social Services, 17, 43 - 62. 